Asia Pac. J. Math. 2022 9:1

ASIA PACIFIC ACADEMIC

\title{
WEIGHTED PSEUDO ALMOST PERIODIC SOLUTION TO PARTIAL STOCHASTIC NEUTRAL EVOLUTION EQUATIONS
}

\author{
MOHSSINE ES-SAIYDY, MOHAMMED ZARHOUNI*, MOHAMED ZITANE
}

Department of Mathematics, MACS Laboratory, Moulay Ismail University, Faculty of Sciences, Meknès, Morocco *Corresponding author: mohammed.zarhouni95@gmail.com

Received Oct. 19, 2021

\begin{abstract}
АвsтRACт. In this paper, we introduce the concept of weighted Stepanov-like square-mean pseudo almost periodic process and study the existence and uniqueness of square-mean almost periodic mild solution for several neutral partial stochastic differential equations with weighted Stepanov-like square-mean pseudo almost periodic coefficient. In addition, an example is provided to illustrate our abstract results.

2010 Mathematics Subject Classification. 34K14, 43A60, 60H15, 47D06.

Key words and phrases. weighted pseudo almost periodic solution; weighted Stepanov-like square-mean pseudo almost periodicity; neutral partial stochastic differential equations; exponentially stable semigroup.
\end{abstract}

\section{INTRODUCTION}

The theory of neutral differential equations is among the most attractive topics in the qualitative theory of differential equations due to their significance and applications in areas such as physics, mathematical biology, control theory, and others, which is why we find them in a lot of work and refer readers to $([12,14-16,20,21])$ and books $([13,19])$. Since stochastic perturbations are unavoidable and omnipresent in nature as well as in man-made systems, we have to move from deterministic models to stochastic models.

The concept of pseudo almost periodicity (p.a.p) was introduced by Zhang ( [24-26]) as a natural generalization of almost periodicity in the sense of $\mathrm{H}$. Bohr. This new concept has aroused great interest among several mathematicians. In 2006, Diagana ( [4]) introduced

DOI: 10.28924/APJM/9-1

()2022 Asia Pacific Journal of Mathematics 
the concept of weighted pseudo almost periodicity, which generalizes the notion of pseudo almost periodicity, and the author proved some interesting properties of the space of weighted pseudo almost periodic functions, like the completeness and the composition theorem, which have many applications in the context of differential equations $([2,5,6,11,27,28])$. Since then, in 2008, Diagana ( [3]) introduced the concept of Stepanov-like pseudo almost periodicity. This concept has undergone several evolutions and has been extended in different directions ( [8-10]). In 2010, Diagana, M. Mophou and M. N'Gurkata( [7]) introduced a new class of functions called weighted Stepanov-like pseudo almost periodic functions, which is the focus of this paper.

In this paper, we study the existence and uniqueness of weighted Stepanov-like square-mean pseudo almost periodic mild solution to the following equation:

$$
d X(t)=A X(t) d t+d g(t, X(t))+h(t, X(t)) d t+f(t, X(t)) d W(t), \quad t \in \mathbb{R} .
$$

Where $A$ is the infinitesimal generator of a $C_{0}$-semigroup on $L^{2}(P, \mathbb{H})$ and $(W(t), t \in \mathbb{R})$ is two-sided standard Weiner process, $h, f$ and $g$ satisfy the hypotheses $\left(A_{1}\right)$ and $\left(A_{2}\right)$ recalled in Section 3.

There are works on the Stepanov pseudo almost periodic solution of equations (1) such as in ( [22]). The purpose of this paper is to deal with the existence and uniqueness of the weighted Stepanov pseudo almost periodic solution of equations (1) which is more general than the above.

The paper is organized as follows, Section 2 is devoted to some basic notations, definitions, and lemmas related to the square-mean pseudo almost periodic process and the weighted pseudo almost periodic process. In Section 3, we study the existence and uniqueness of weighted pseudo almost periodic solution to the neutral partial stochastic differential equation (1), and in Section 4, we present an example to illustrate the abstract result.

\section{Preliminary}

In this section, we present some basic notation and collect some necessary definitions and lemmas which will be used in thereafter. Throughout this paper:

- $\left(\mathbb{K},\|\cdot\|_{\mathbb{K}}\right)$ and $\left(\mathbb{H},\|\cdot\|_{\mathbb{H}}\right)$ are real separable Hilbert spaces.

- $(\Omega, \mathcal{F}, P)$ is a complete probability space. 
- $L^{2}(P, \mathbb{H})$ is a space off all $\mathbb{H}$-valued random variable $X$ is a Banach space with the norm $\|X\|_{2}=\left(\int_{\Omega}\|X\|^{2} d P\right)^{\frac{1}{2}}$ and it is common to verify that $L^{2}(P, \mathbb{H})$ is a Hilbert space with the norm $\|$.$\| .$

- $L(\mathbb{K}, \mathbb{H})$ represent the space of all linear bounded operators from $\mathbb{K}$ into $\mathbb{H}$ equipped with the usual operator norm $\|\cdot\|_{L(\mathbb{K}, \mathbb{H})}$.

- $A$ is a the infinitesimal generator of an exponentially stable $C_{0}$-semigroup $\{T(t)\}_{t \geqslant 0}$ on $L^{2}(P, \mathbb{H})$, i.e., there exist positive constants $M, \delta>0$ such that

$$
\|T(t)\| \leq M e^{-\delta t}, \quad \forall t \geq 0
$$

- $(W(t), t \in \mathbb{R})$ is a two-sided standard Weiner process defined on the filtered probability $\operatorname{space}\left(\Omega, \mathcal{F},\left(\mathcal{F}_{t}\right)_{t \geq 0}, P\right)$ where $\mathcal{F}_{t}=\sigma\{W(a)-W(b) ; a, b \leq t\}$.

Definition 2.1 ( $[23])$. A stochastic process $X: \mathbb{R} \rightarrow L^{2}(P, \mathbb{H})$ is said to be stochastically continuous if

$$
\lim _{t \rightarrow s} E\|X(t)-X(s)\|^{2}=0
$$

Definition 2.2 ( $[23])$. A stochastic process $X: \mathbb{R} \rightarrow L^{2}(P, \mathbb{H})$ is said to be stochastically bounded if there exists $M \geq 0$, such that $E\|X(t)\|^{2} \leqslant M$. The collection of all stochastically bounded and continuous processes is then denoted by $B C\left(\mathbb{R}, L^{2}(P, \mathbb{H})\right)$.

Definition 2.3 ( $[23]$ ). A continuous stochastic process $X: \mathbb{R} \rightarrow L^{2}(P, \mathbb{H})$ is called squaremean almost periodic if for each $\varepsilon>0$ there exists $\ell(\varepsilon)>0$ such that every interval of length $\ell(\varepsilon)$ contains at least a number $r$ with the property that

$$
\sup _{t \in \mathbb{R}} E\|X(t+r)-X(t)\|^{2}<\varepsilon
$$

The space of all such functions will be denoted by $A P\left(\mathbb{R}, L^{2}(P, \mathbb{H})\right)$.

Definition 2.4 ( [23]). A jointly continuous process $F: \mathbb{R} \times L^{2}(P, \mathbb{K}) \rightarrow L^{2}(P, \mathbb{H})$ is called almost periodic in $t \in \mathbb{R}$ uniformly in $X \in L^{2}(P, \mathbb{K})$ if for each $\varepsilon>0$ and any compact $K \subset L^{2}(P, \mathbb{K})$ there exists $\ell(\varepsilon)$ such that every interval of length $\ell(\varepsilon)$ contains a number $\tau$ with the property that

$$
E\|F(t+\tau, X)-F(t, X)\|^{2}<\varepsilon
$$

The collection of those functions is denoted by $A P\left(\mathbb{R} \times L^{2}(P, \mathbb{K}), L^{2}(P, \mathbb{H})\right)$. 
Let $\mathcal{U}$ be the collection of functions (weights) $\rho: \mathbb{R} \rightarrow(0, \infty)$ which are locally integrable over $\mathbb{R}$ with $\rho>0$, for $T>0$, we denote

$$
m(T, \rho)=\int_{-T}^{T} \rho(x) d x
$$

Define

$$
\mathcal{U}_{\infty}:=\left\{\rho \in \mathcal{U}: \lim _{T \rightarrow \infty} m(T, \rho)=\infty\right\}
$$

And

$$
\mathcal{U}_{b}:=\left\{\rho \in \mathcal{U}_{\infty}: \rho \text { is bounded and } \inf _{x \in \mathbb{R}} \rho(x)>0\right\} .
$$

Now, for $\rho \in \mathcal{U}_{\infty}$ we define

$$
P A P_{0}\left(\mathbb{R}, L^{2}(P, \mathbb{H}), \rho\right):=\left\{X \in B C\left(\mathbb{R}, L^{2}(P, \mathbb{H})\right): \lim _{T \rightarrow \infty} \frac{1}{m(T, \rho)} \int_{-T}^{T} E\|X(t)\|^{2} \rho(t) d t=0\right\} .
$$

Similarly, we define $P A P_{0}\left(\mathbb{R} \times L^{2}(P, \mathbb{K}), L^{2}(P, \mathbb{H}), \rho\right)$ as the collection of jointly continuous process $F: \mathbb{R} \times L^{2}(P, \mathbb{K}) \rightarrow L^{2}(P, \mathbb{H})$ such that $F(., Q)$ is bounded for each $Q \in L^{2}(P, \mathbb{K})$ and

$$
\lim _{T \rightarrow \infty} \frac{1}{m(T, \rho)} \int_{-T}^{T} E\|F(s, X)\|^{2} \rho(s) d s=0
$$

uniformly in $Q \in L^{2}(P, \mathbb{K})$.

Definition 2.5 ( $[4])$. A stochastic process $X \in B C\left(\mathbb{R}, L^{2}(P, \mathbb{H})\right)$ is called weighted pseudo almost periodic (or $\rho$-pseudo almost periodic) if it can be expressed as $X=Y+Z$, where $Y \in A P\left(\mathbb{R}, L^{2}(P, \mathbb{H})\right)$ and $Z \in P A P_{0}\left(\mathbb{R}, L^{2}(P, \mathbb{H}), \rho\right)$. The collection of such functions will be denoted by $P A P\left(\mathbb{R}, L^{2}(P, \mathbb{H}), \rho\right)$.

Definition 2.6 ( $[4])$. A stochastic process $F \in C\left(\mathbb{R} \times L^{2}(P, \mathbb{K}), L^{2}(P, \mathbb{H})\right)$ is called weighted pseudo almost periodic in $t \in \mathbb{R}$ uniformly in $Q \in L^{2}(P, \mathbb{K})$ if it can be expressed as $F=G+H$, where $G \in A P\left(\mathbb{R} \times L^{2}(P, \mathbb{K}), L^{2}(P, \mathbb{H})\right)$ and $H \in P A P_{0}\left(\mathbb{R} \times L^{2}(P, \mathbb{K}), L^{2}(P, \mathbb{H}), \rho\right)$. The collection of such functions will be denoted by $P A P\left(\mathbb{R} \times L^{2}(P, \mathbb{K}), L^{2}(P, \mathbb{H}), \rho\right)$.

Lemma $2.7([1])$. If $\rho \in \mathcal{U}_{\infty}$ and if the limits

$$
\lim _{t \rightarrow \infty} \frac{\rho(t+r)}{t} \text { and } \lim _{t \rightarrow \infty} \frac{m(t+r, \rho)}{m(t, \rho)}
$$

exist for all $r \in \mathbb{R}$. Then, $P A P\left(\mathbb{R}, L^{2}(P, \mathbb{H}), \rho\right)$ is translation-invariant. The collection of all weights $\rho \in \mathcal{U}_{\infty}$ such that $P A P\left(\mathbb{R}, L^{2}(P, \mathbb{H}), \rho\right)$ is translation-invariant is denoted by $\mathcal{U}_{\infty}^{\text {inv }}$. 


\section{Main Results}

This section focuses on the important properties of stochastic weighted pseudo almost periodic functions and introduces a new class of functions called weighted Stepanov-like square-mean pseudo almost periodic functions and investigates its properties.

Throughout this paper, we fix $\rho \in \mathcal{U}_{\infty}$ such that

$$
\lim _{t \rightarrow \infty} \frac{\rho(t+r)}{t}<\infty
$$

and,

$$
\lim _{t \rightarrow \infty} \frac{m(t+r, \rho)}{m(t, \rho)}<\infty
$$

Lemma 3.1. If $X=Y+Z$, where $X \in P A P\left(\mathbb{R}, L^{2}(P, \mathbb{H}), \rho\right)$ and $Y \in A P\left(\mathbb{R}, L^{2}(P, \mathbb{H})\right)$ and $Z \in P A P_{0}\left(\mathbb{R}, L^{2}(P, \mathbb{H}), \rho\right)$ then

$$
Y(\mathbb{R}) \subset \overline{X(\mathbb{R})} \text { and } E\|X\|^{2} \geq E\|Y\|^{2}
$$

Proof. By absurd we suppose that $Y(\mathbb{R}) \nsubseteq \overline{X(\mathbb{R})}$.

Then, there exist $t_{1} \in \mathbb{R}$ and $\varepsilon_{1}>0$ such that

$$
\inf _{x \in \mathbb{R}} E\left\|Y\left(t_{1}\right)-X(x)\right\|^{2}>\varepsilon_{1} .
$$

Since $Y \in A P\left(\mathbb{R},{ }^{2}(P, \mathbb{H})\right)$, then there exist $\ell_{1}\left(\varepsilon_{1}\right)$ such that every interval of length $\ell_{1}\left(\varepsilon_{1}\right)$ contains a number $\tau$

$$
E\|Y(t+\tau)-Y(t)\|^{2}<\frac{\varepsilon_{1}}{4} .
$$

We have $Y$ is a continuous process, then there exists $\alpha>0$ such that for all $t \in\left(t_{1}-\alpha, t_{1}+\alpha\right)$

$$
E\left\|Y(t)-Y\left(t_{1}\right)\right\|^{2}<\frac{\varepsilon_{1}}{2} .
$$

We know that $P A P\left(\mathbb{R}, L^{2}(P, \mathbb{H}), \rho\right)$ is translation invariant, then, $Z(t+$.) $\in$ $P A P_{0}\left(\mathbb{R}, L^{2}(P, \mathbb{H}), \rho\right)$.

Which implies that for all $t \in\left(t_{1}-\alpha, t_{1}+\alpha\right)$ and for every interval of length $\ell_{1}\left(\varepsilon_{1}\right)$ contains a number $\tau$

$$
\begin{aligned}
E\|Z(t+\tau)\|^{2} & =E\|X(t+\tau)-Y(t+\tau)\|^{2}, \\
& \geqslant E\|X(t+\tau)-Y(t)\|^{2}-E\|Y(t)-Y(t+\tau)\|^{2}, \\
& \geqslant E\|X(t+\tau)-Y(t)\|^{2}-E\left\|Y(t)-Y\left(t_{1}\right)\right\|^{2}-E\|Y(t)-Y(t+\tau)\|^{2},
\end{aligned}
$$




$$
\begin{aligned}
& \geqslant \inf _{x \in \mathbb{R}} E\left\|X(x)-Y\left(t_{1}\right)\right\|^{2}-E\left\|Y(t)-Y\left(t_{1}\right)\right\|^{2}-E\|Y(t)-Y(t+\tau)\|^{2}, \\
& \geqslant \varepsilon_{1}-\frac{\varepsilon_{1}}{2}-\frac{\varepsilon_{1}}{4} \\
& \geqslant \frac{\varepsilon_{1}}{4} .
\end{aligned}
$$

Thus, $\lim _{T \rightarrow \infty} \frac{1}{m(T, \rho)} \int_{-T}^{T} E\|Z(t+\tau)\|^{2} \rho(t) d t>0$, which is absurd with the fact that $Z(t+.) \in$ $P A P_{0}\left(\mathbb{R}, L^{2}(P, \mathbb{H}), \rho\right)$.

Lemma 3.2. If $\left\{X_{n}\right\}_{n \in \mathbb{N}} \subset P A P\left(\mathbb{R}, L^{2}(P, \mathbb{H}), \rho\right)$ be a sequence of stochastic process and if $X_{n}$ converge uniformly to $X$, then $X \in P A P\left(\mathbb{R}, L^{2}(P, \mathbb{H}), \rho\right)$.

Proof. Let $X_{n}=Y_{n}+Z_{n}$ where $\left\{Y_{n}\right\}_{n \in \mathbb{N}} \subset A P\left(\mathbb{R}, L^{2}(P, \mathbb{H})\right)$ and $\left\{Z_{n}\right\}_{n \in \mathbb{N}} \subset$ $P A P_{0}\left(\mathbb{R}, L^{2}(P, \mathbb{H}), \rho\right)$. From Lemma(3.1) we have $E\left\|X_{n}\right\|^{2} \geq E\left\|Y_{n}\right\|^{2}$ and there exist $Y \in A P\left(\mathbb{R}, L^{2}(P, \mathbb{H})\right)$ such that $\left\|Y_{n}-Y\right\|_{\infty} \rightarrow 0$ as $n \rightarrow \infty$.

By using the preceding fact, there exists a function $Z \in B C\left(\mathbb{R}, L^{2}(P, \mathbb{H})\right)$ such that

$$
\left\|Z_{n}-Z\right\|_{\infty} \rightarrow 0 \text { as } n \rightarrow \infty
$$

Now, for $T>0$

$$
\begin{aligned}
\frac{1}{m(T, \rho)} \int_{-T}^{T} E\|Z\|^{2} \rho(t) d t & \leqslant \frac{1}{m(T, \rho)} \int_{-T}^{T} E\left\|Z_{n}-Z\right\|^{2} \rho(t) d t \\
& +\frac{1}{m(T, \rho)} \int_{-T}^{T} E\left\|Z_{n}\right\|^{2} \rho(t) d t \\
& \leqslant E\left\|Z_{n}-Z\right\|^{2}+\frac{1}{m(T, \rho)} \int_{-T}^{T} E\left\|Z_{n}\right\|^{2} \rho(t) d t .
\end{aligned}
$$

So, $Z \in P A P_{0}\left(\mathbb{R}, L^{2}(P, \mathbb{H}), \rho\right)$.

Finally, $X=Y+Z \in P A P\left(\mathbb{R}, L^{2}(P, \mathbb{H}), \rho\right)$.

Lemma 3.3. Suppose that $Q(.) \in P A P\left(\mathbb{R}, L^{2}(P, \mathbb{K}), \rho\right), F \in P A P\left(\mathbb{R} \times L^{2}(P, \mathbb{K}), L^{2}(P, \mathbb{H}), \rho\right)$ and there exist a positive number $L$ such that for any $X, Y \in L^{2}(P, \mathbb{K})$

$$
E\|F(t, X)-F(t, Y)\|^{2} \leqslant L E\|X-Y\|^{2}, \quad \forall t \in \mathbb{R}
$$

Then, $F(., Q().) \in P A P\left(\mathbb{R}, L^{2}(P, \mathbb{H}), \rho\right)$.

Remark 3.4. Lemma (3.3) it is a generalization of the known composition results of pseudo almost periodic functions, one can refer to ( [26]).

Lemma 3.5. Let $X \in P A P\left(\mathbb{R}, L^{2}(P, \mathbb{H}), \rho\right)$ and $\Lambda \in \mathcal{L}\left(L^{2}(P, \mathbb{H}), L^{2}(P, \mathbb{K})\right)$. If $Y(t)=\Lambda X(t)$, then $Y \in P A P\left(\mathbb{R}, L^{2}(P, \mathbb{K}), \rho\right)$. 
Proof. The proof of Lemma (3.5) is similar to the proof of Lemma (4.1) in ( [17]).

Now, we define the space of weighted Stepanov-like square-mean pseudo almost periodic functions.

Definition 3.6. Let $t \in \mathbb{R}, s \in[0.1]$ and $X \in L^{2}(P, \mathbb{K})$.

i) We define the Bochner transform $G^{b}(t, s)$ of a stochastic process $X: \mathbb{R} \rightarrow L^{2}(P, \mathbb{H})$ by $G^{b}(t, s):=G(t+s)$.

ii) We define the Bochner transform $F^{b}(t, s, X)$ of a stochastic process $F: \mathbb{R} \times L^{2}(P, \mathbb{K}) \rightarrow$ $L^{2}(P, \mathbb{H})$ by $F^{b}(t, s, X):=F(t+s, X)$.

Remark 3.7. A stochastic process $Y(t, s)$ is the Bochner transform of a certain stochastic process $X$

$$
Y(t, s)=X^{b}(t, s)
$$

if and only if

$$
Y(t+\tau, s-\tau)=Y(t, s) \quad \forall \tau \in[s-1, s]
$$

Definition 3.8. The space $B S^{2}\left(\mathbb{R}, L^{2}(P, \mathbb{H})\right)$ of all stepanov bounded stochastic processes consists of all measurable stochastic processes $X: \mathbb{R} \rightarrow L^{2}(P, \mathbb{H})$ such that

$$
X^{b} \in L^{\infty}\left(\mathbb{R}, L^{2}\left((0,1), L^{2}(P, \mathbb{H})\right)\right)
$$

This is a Banach space with the norm

$$
\|X\|_{S^{2}}=\left\|X^{b}\right\|_{L^{\infty}\left(\mathbb{R}, L^{2}\right)}=\sup _{t \in \mathbb{R}}\left(\int_{t}^{t+1} E\|X(s)\|^{2} d s\right)^{\frac{1}{2}} .
$$

Definition 3.9. A stochastic process $X \in B S^{2}\left(\mathbb{R}, L^{2}(P, \mathbb{H})\right)$ is said to be a $S^{2}$-weighted pseudo almost periodic (or weighted Stepanov-like square-mean pseudo almost periodic) if it can be expressed as $X=Y+Z$, where $Y^{b} \in A P\left(\mathbb{R}, L^{2}\left((0,1), L^{2}(P, \mathbb{H})\right)\right)$ and $Z^{p} \in$ $P A P_{0}\left(\mathbb{R}, L^{2}\left((0,1), L^{2}(P, \mathbb{H})\right), \rho\right)$.

The collection of such functions will be denoted by $P A P S^{2}\left(\mathbb{R}, L^{2}(P, \mathbb{H}), \rho\right)$.

Definition 3.10. A stochastic process $F \in B S^{2}\left(\mathbb{R} \times L^{2}(P, \mathbb{K}), L^{2}(P, \mathbb{H})\right)$ is said to be Stepanovlike square-mean pseudo almost periodic if it can be decomposed as $F=X+Y$ where $X^{b} \in$ $A P\left(\mathbb{R} \times L^{2}(P, \mathbb{K}), L^{2}\left((0,1), L^{2}(P, \mathbb{H})\right)\right)$ and $Y^{b} \in P A P_{0}\left(\mathbb{R} \times L^{2}(P, \mathbb{K}), L^{2}\left((0,1), L^{2}(P, \mathbb{H})\right), \rho\right)$. The space of all such functions will be denoted by $P A P S^{2}\left(\mathbb{R} \times L^{2}(P, \mathbb{K}), L^{2}(P, \mathbb{H}), \rho\right)$. 
Lemma 3.11. Let $Q(.) \in P A P S^{2}\left(\mathbb{R}, L^{2}(P, \mathbb{K}), \rho\right), F \in P A P S^{2}\left(\mathbb{R} \times L^{2}(P, \mathbb{K}), L^{2}(P, \mathbb{H})\right.$, $\left.\rho\right)$ and there exist $L \geqslant 0$ such that

$$
E\|F(t, X)-F(t, Y)\|^{2} \leqslant L E\|X-Y\|^{2}, \quad \forall X, Y \in L^{2}(\mathbb{P}, \mathbb{K}) .
$$

Then, $F(., Q()$.$) belongs to P A P S^{2}\left(\mathbb{R}, L^{2}(P, \mathbb{H}), \rho\right)$.

Proof. Let $F=F_{1}+F_{2}$, where $F_{1}^{b} \in A P\left(\mathbb{R} \times L^{2}(P, \mathbb{K}), L^{2}\left((0,1), L^{2}(P, \mathbb{H})\right)\right)$ and $F_{2}^{b} \in P A P_{0}(\mathbb{R} \times$ $\left.L^{2}(P, \mathbb{K}), L^{2}\left((0,1), L^{2}(P, \mathbb{H})\right), \rho\right)$, let $\Phi=\Phi_{1}+\Phi_{2}$ where $\Phi_{1}^{b} \in A P\left(\mathbb{R}, L^{2}\left((0,1), L^{2}(P, \mathbb{H})\right)\right)$ and $\Phi_{2}^{b} \in P A P_{0}\left(\mathbb{R}, L^{2}\left((0,1), L^{2}(P, \mathbb{H})\right), \rho\right)$, we can decompose $F^{b}$ as follows

$$
\begin{aligned}
F^{b}\left(., \Phi^{b}(.)\right) & =F_{1}^{b}\left(., \Phi_{1}^{b}(.)\right)+F^{b}\left(., \Phi^{b}(.)\right)-F_{1}^{b}\left(., \Phi_{1}^{b}(.)\right) \\
& =F_{1}^{b}\left(., \Phi_{1}^{b}(.)\right)+F^{b}\left(., \Phi^{b}(.)\right)-F^{b}\left(., \Phi_{1}^{b}(.)\right)+F_{2}^{b}\left(., \Phi_{1}^{b}(.)\right) .
\end{aligned}
$$

By using Lemma (3.3) it is easy to see that $F_{1}^{b}\left(., \Phi_{1}^{b}().\right) \in P A P\left(\mathbb{R}, L^{2}\left((0,1), L^{2}(P, \mathbb{H})\right), \rho\right)$ and $F_{2}^{b}\left(., \Phi_{1}^{b}().\right) \in P A P_{0}\left(\mathbb{R}, L^{2}\left((0,1), L^{2}(P, \mathbb{H})\right), \rho\right)$.

Now, set

$$
H^{b}(.)=F^{b}\left(., \Phi^{b}(.)\right)-F_{1}^{b}\left(., \Phi_{1}^{b}(.)\right),
$$

we have

$$
\begin{aligned}
\int_{t}^{t+1} E\|H(r)\|^{2} d r & =\int_{t}^{t+1} E\left\|F(r, \Phi(r))-F_{1}\left(r, \Phi_{1}(r)\right)\right\|^{2} \\
& \leq L^{2} \int_{t}^{t+1} E\left\|\Phi(r)-\Phi_{1}(r)\right\|^{2} d r, \\
& \leq L^{2} \int_{t}^{t+1} E\left\|\Phi_{2}(r)\right\|^{2} d r,
\end{aligned}
$$

which implies that

$\lim _{T \rightarrow \infty} \frac{1}{m(T, \rho)} \int_{-T}^{T}\left(\int_{t}^{t+1} E\|H(r)\|^{2} d r\right)^{\frac{1}{2}} \rho(t) d t \leq \lim _{T \rightarrow \infty} \frac{L}{m(T, \rho)} \int_{-T}^{T}\left(\int_{t}^{t+1} E\left\|\Phi_{2}(r)\right\|^{2} d r\right)^{\frac{1}{2}} \rho(t) d t$.

We know that $\Phi_{2}^{b} \in P A P_{0}\left(\mathbb{R}, L^{2}\left((0,1), L^{2}(P, \mathbb{H})\right), \rho\right)$, which means that for all $t \in \mathbb{R}$

$$
\lim _{T \rightarrow \infty} \frac{1}{m(T, \rho)} \int_{-T}^{T}\left(\int_{t}^{t+1} E\left\|\Phi_{2}(r)\right\|^{2} d r\right)^{\frac{1}{2}} \rho(t) d t=0 .
$$

Therefore,

$$
\lim _{T \rightarrow \infty} \frac{1}{m(T, \rho)} \int_{-T}^{T}\left(\int_{t}^{t+1} E\|H(r)\|^{2} d r\right)^{\frac{1}{2}} \rho(t) d t=0 .
$$

Hence, $H^{b} \in P A P_{0}\left(\mathbb{R}, L^{2}\left((0,1), L^{2}(P, \mathbb{H})\right), \rho\right)$. Finally, we conclude that $F(., Q()$.$) belongs to$ $P A P S^{2}\left(\mathbb{R}, L^{2}(P, \mathbb{H}), \rho\right)$. 
Lemma 3.12. Let $X \in P A P S^{2}\left(\mathbb{R}, L^{2}(P, \mathbb{H}), \rho\right)$ and $\Lambda \in \mathcal{L}\left(L^{2}(P, \mathbb{H}), L^{2}(P, \mathbb{K})\right)$, if $Y(t)=\Lambda X(t)$, then $Y \in P A P S^{2}\left(\mathbb{R}, L^{2}(P, \mathbb{K}), \rho\right)$.

Proof. We have $X \in B S^{2}\left(\mathbb{R}, L^{2}(P, \mathbb{H})\right)$ and $\Lambda \in \mathcal{L}\left(L^{2}(P, \mathbb{H}), L^{2}(P, \mathbb{K})\right)$, then $Y \quad \in$ $B S^{2}\left(\mathbb{R}, L^{2}(P, \mathbb{H})\right)$. Let $X=X_{1}+X_{2}$, we get $X^{b}=X_{1}^{b}+X_{2}^{b}$, where $X_{1}^{b} \in$ $A P\left(\mathbb{R}, L^{2}\left((0,1), L^{2}(P, \mathbb{H})\right)\right)$ and $X_{2}^{b} \in P A P_{0}\left(\mathbb{R}, L^{2}\left((0,1), L^{2}(P, \mathbb{H})\right), \rho\right)$. For $t \in \mathbb{R}$ and $s \in[0,1]$, we set

$$
\begin{aligned}
Y(t+s) & =\Lambda X_{1}(t+s)+\Lambda X_{2}(t+s) \\
& =Y_{1}(t+s)+Y_{2}(t+s)
\end{aligned}
$$

Then,

$$
\begin{aligned}
Y^{b}(t+s) & =Y_{1}^{b}(t, s)+Y_{2}^{b}(t, s) \\
& =\Lambda X_{1}^{b}(t, s)+\Lambda X_{2}^{b}(t, s) .
\end{aligned}
$$

By Lemma (3.5), we get $Y_{1}^{b} \in \quad \in A P\left(\mathbb{R}, L^{2}\left((0,1), L^{2}(P, \mathbb{K})\right)\right)$ and $Y_{2}^{b} \in$ $P A P_{0}\left(\mathbb{R}, L^{2}\left((0,1), L^{2}(P, \mathbb{K})\right)\right)$. So, $Y \in P A P S^{2}\left(\mathbb{R}, L^{2}(P, \mathbb{K}), \rho\right)$.

\section{Weighted Pseudo Almost Periodic Solution}

In this section, we establish the existence and uniqueness of pseudo almost periodic solution to partial stochastic neutral differential equations with weighted Stepanov-like square-mean pseudo almost periodic coefficients (1).

We will list a few hypotheses which will be used for the rest of this paper.

$\left(\mathbb{A}_{1}\right)$ : The functions $h, f \in P A P S^{2}\left(\mathbb{R} \times L^{2}(P, \mathbb{H}), \rho, L^{2}(P, \mathbb{H})\right) \cap C\left(\mathbb{R} \times L^{2}(P, \mathbb{H}), L^{2}(P, \mathbb{H})\right)$.

$\left(\mathbb{A}_{2}\right)$ : The function $g \in P A P\left(\mathbb{R} \times L^{2}(P, \mathbb{H}), L^{2}(P, \mathbb{H}), \rho\right) \cap C\left(\mathbb{R} \times L^{2}(P, \mathbb{H}), L^{2}(P, \mathbb{H})\right)$.

$\left(\mathbb{A}_{3}\right)$ : There exist positive constants $K_{g}, K_{h}, K_{f}>0$ such that for any stochastic process $X, Y \in L^{2}(P, \mathbb{H})$, the functions $g, h, f$ satisfy

$$
\begin{aligned}
& E\|g(t, X)-g(t, Y)\|^{2} \leqslant K_{g} E\|X-Y\|^{2} . \\
& E\|h(t, X)-h(t, Y)\|^{2} \leqslant K_{h} E\|X-Y\|^{2} . \\
& E\|f(t, X)-f(t, Y)\|^{2} \leqslant K_{f} E\|X-Y\|^{2} .
\end{aligned}
$$


Definition 4.1. Let $X: \mathbb{R} \rightarrow{ }^{2}(P, \mathbb{H})$, a $\mathcal{F}_{t}$ progressively measurable stochastic process $X$ is called mild solution of the equation $(1)$ if $X(t)$ satisfies

$$
\begin{aligned}
X(t)-g(t, X(t)) & =T(t-s)[X(s)-g(s, X(s))] \\
& +\int_{s}^{t} T(t-\tau) h(\tau) d \tau+\int_{s}^{t} T(t-\tau) f(\tau) d W(\tau), \quad \forall t \geq s
\end{aligned}
$$

Lemma 4.2. Let $h \in P A P S^{2}\left(\mathbb{R}, L^{2}(P, \mathbb{H}), \rho\right) \cap C\left(\mathbb{R}, L^{2}(P, \mathbb{H})\right)$. Consider the function $H$ defined by

$$
H(t):=\int_{-\infty}^{t} T(t-\tau) h(\tau) d \tau, \quad \forall t \in \mathbb{R}
$$

Then $H \in P A P\left(\mathbb{R}, L^{2}(P, \mathbb{H}), \rho\right)$.

Proof. Since $h \in P A P S^{2}\left(\mathbb{R}, L^{2}(P, \mathbb{H}), \rho\right) \cap C\left(\mathbb{R}, L^{2}(P, \mathbb{H})\right)$, write $h=h_{1}+h_{2}$, where

$$
h_{1}^{b} \in A P\left(\mathbb{R}, L^{2}\left((0,1), L^{2}(P, \mathbb{H})\right)\right) \cap C\left(\mathbb{R}, L^{2}\left((0,1), L^{2}(P, \mathbb{H})\right)\right)
$$

And

$$
h_{2}^{b} \in P A P_{0}\left(\mathbb{R}, L^{2}\left((0,1), L^{2}(P, \mathbb{H})\right), \rho\right) \cap C\left(\mathbb{R}, L^{2}\left((0,1), L^{2}(P, \mathbb{H})\right)\right)
$$

Then, $H(t)$ can be decomposed as $H(t)=G(t)+\Phi(t)$ such that $G(t)=\int_{-\infty}^{t} T(t-\tau) h_{1}(\tau) d \tau$ and $\Phi(t)=\int_{-\infty}^{t} T(t-\tau) h_{2}(\tau) d \tau$.

By similar proof to that of Lemma 3.1 in ( [22]), one can obtain that $G(.) \in A P\left(\mathbb{R}, L^{2}(P, \mathbb{H})\right)$.

Let us show that $\Phi(.) \in P A P_{0}\left(\mathbb{R}, L^{2}(P, \mathbb{H}), \rho\right)$.

Define for each $i=1,2,3, \ldots$

$$
\Phi_{i}(t)=\int_{i-1}^{i} T(\tau) h_{2}(t-\tau) d \tau=\int_{t-i}^{t-i+1} T(t-\tau) h_{2}(\tau) d \tau, \quad \forall t \in \mathbb{R}
$$

Thus,

$$
\begin{aligned}
E\left\|\Phi_{i}(t)\right\|^{2} & =E\left\|\int_{t-i}^{t-i+1} T(t-\tau) h_{2}(\tau) d \tau\right\|^{2} \\
& \leq E\left(\int_{t-i}^{t-i+1}\|T(t-\tau)\|\left\|h_{2}(\tau)\right\| d \tau\right)^{2} .
\end{aligned}
$$

By using the exponential stable of $T(t)_{t \geq 0}$, we have

$$
E\left\|\Phi_{i}(t)\right\|^{2} \leq E\left(\int_{t-i}^{t-i+1} M e^{-\delta(t-\tau)}\left\|h_{2}(\tau)\right\| d \tau\right)^{2} .
$$


By using Hölder inequality we have

$$
\begin{aligned}
E\left\|\Phi_{i}(t)\right\|^{2} & \leq M^{2}\left(\int_{t-i}^{t-i+1} e^{-2 \delta(t-\tau)} d \tau\right)\left(\int_{t-i}^{t-i+1} E\left\|h_{2}(\tau)\right\|^{2} d \tau\right) \\
& \leq M^{2}\left(\int_{i}^{i-1} e^{-2 \delta \tau} d \tau\right)\left(\int_{t-i}^{t-i+1} E\left\|h_{2}(\tau)\right\|^{2} d \tau\right) \\
& \leq \frac{M^{2}}{2 \delta}\left(e^{2 \delta}-1\right) e^{-2 \delta i}\left(\int_{t-i}^{t-i+1} E\left\|h_{2}(\tau)\right\|^{2} d \tau\right) \\
& \leq \frac{M^{2}}{2 \delta}\left(e^{2 \delta}-1\right) e^{-2 \delta i}\left\|h_{2}\right\|_{S^{2}}^{2}
\end{aligned}
$$

As a consequence, we get from the Well-Known Weierstrass test that the series $\sum_{i=1}^{\infty} \Phi_{i}(t)$ is uniformly convergent on $\mathbb{R}$, what is more

$$
\Phi(t)=\int_{-\infty}^{t} T(\tau) h_{2}(t-\tau) d \tau=\sum_{i=1}^{\infty} \Phi_{i}(t) \in B C\left(\mathbb{R}, L^{2}(\mathbb{P}, \mathbb{H})\right)
$$

Now, let's shows that

$$
\lim _{T \rightarrow \infty} \frac{1}{m(T, \rho)} \int_{-T}^{T} E\left\|\Phi_{i}(t)\right\|^{2} \rho(t) d t=0
$$

We have

$$
\begin{array}{rl}
\lim _{T \rightarrow \infty} \frac{1}{m(T, \rho)} \int_{-T}^{T} & E\left\|\Phi_{i}(t)\right\|^{2} \rho(t) d t \\
& \leq \frac{M^{2}}{2 \delta}\left(e^{2 \delta}-1\right) e^{-2 \delta i} \lim _{T \rightarrow \infty} \frac{1}{m(T, \rho)} \int_{-T}^{T} \int_{t-i}^{t-i+1} E\left\|h_{2}(\tau)\right\|^{2} d \tau, \\
& \leq \frac{M^{2}}{2 \delta}\left(e^{2 \delta}-1\right) e^{-2 \delta i} \lim _{T \rightarrow \infty} \frac{1}{m(T, \rho)} \int_{-T}^{T}\left\|h_{2}^{b}(t-i)\right\|_{L^{\infty}\left(\mathbb{R}, L^{2}\right)} d t .
\end{array}
$$

Since $h_{2}^{b}(.) \in P A P_{0}\left(\mathbb{R}, L^{2}(P, \mathbb{H}), \rho\right)$, then

$$
\lim _{T \rightarrow \infty} \frac{1}{m(T, \rho)} \int_{-T}^{T}\left\|h_{2}^{b}(t-i)\right\|_{L^{\infty}\left(\mathbb{R}, L^{2}\right)} d t=0 .
$$

Therefore, $\Phi_{i}(.) \in P A P_{0}\left(\mathbb{R}, L^{2}(P, \mathbb{H}), \rho\right)$. By Lemma (3.2), we get $\Phi(.) \in P A P_{0}\left(\mathbb{R}, L^{2}(P, \mathbb{H}), \rho\right)$. Finally, $H(.) \in P A P\left(\mathbb{R}, L^{2}(P, \mathbb{H}), \rho\right)$.

Lemma 4.3. Let $f \in P A P S^{2}\left(\mathbb{R}, L^{2}(P, \mathbb{H}), \rho\right) \cap C\left(\mathbb{R}, L^{2}(P, \mathbb{H})\right)$. Consider the function $F$ defined by

$$
F(t):=\int_{-\infty}^{t} T(t-\tau) f(\tau) d W(\tau), \forall t \in \mathbb{R}
$$

Then, $F \in P A P\left(\mathbb{R}, L^{2}(P, \mathbb{H}), \rho\right)$. 
Proof. Since $f \in P A P S^{2}\left(\mathbb{R}, L^{2}(P, \mathbb{H}), \rho\right) \cap C\left(\mathbb{R}, L^{2}(P, \mathbb{H})\right)$, we can write $f=f_{1}+f_{2}$, where

$$
f_{1}^{b} \in A P\left(\mathbb{R}, L^{2}\left((0,1), L^{2}(P, \mathbb{H})\right)\right) \cap C\left(\mathbb{R}, L^{2}\left((0,1), L^{2}(P, \mathbb{H})\right)\right)
$$

And

$$
f_{2}^{b} \in P A P_{0}\left(\mathbb{R}, L^{2}\left((0,1), L^{2}(P, \mathbb{H})\right), \rho\right) \cap C\left(\mathbb{R}, L^{2}\left((0,1), L^{2}(P, \mathbb{H})\right)\right)
$$

Then, $F(t)$ can be decomposed as $F(t)=X(t)+Y(t)$ such that $X(t)=\int_{-\infty}^{t} T(t-\tau) f_{1}(\tau) d W(\tau)$ and $Y(t)=\int_{-\infty}^{t} T(t-\tau) f_{2}(\tau) d W(\tau)$.

By similar proof to that of Lemma 3.2 in $([22])$, one can obtain that $X(.) \in A P\left(\mathbb{R}, L^{2}(P, \mathbb{H})\right)$. Let us show that $Y(.) \in P A P_{0}\left(\mathbb{R}, L^{2}(P, \mathbb{H}), \rho\right)$. Define for each $i=1,2,3, \ldots$

$$
Y_{i}(t)=\int_{i-1}^{i} T(\tau) f_{2}(t-\tau) d W(\tau)=\int_{t-i}^{t-i+1} T(t-\tau) f_{2}(\tau) d W(\tau), \forall t \in \mathbb{R} .
$$

By using an estimate on the Ito integral ( [18]), we have

$$
\begin{aligned}
E\left\|Y_{i}(t)\right\|^{2} & =E\left\|\int_{t-i}^{t-i+1} T(t-\tau) f_{2}(\tau) d W(\tau)\right\|^{2} \\
& \leq \int_{t-i}^{t-i+1}\|T(t-\tau)\|^{2} E\left\|f_{2}(\tau)\right\|^{2} d \tau
\end{aligned}
$$

By using the exponential stable of $T(t)_{t \geq 0}$ we have

$$
\begin{aligned}
E\left\|Y_{i}(t)\right\|^{2} & \leq M^{2} \int_{t-i}^{t-i+1} e^{-\delta(t-\tau)} E\left\|f_{2}(\tau)\right\|^{2} d \tau \\
& \leq M^{2} \int_{i-1}^{i} e^{-2 \delta \tau} E\left\|f_{2}(t-\tau)\right\|^{2} d \tau \\
& \leq M^{2} \sup _{\tau \in[i-1, i]} e^{-2 \delta \tau} \int_{i-1}^{i} E\left\|f_{2}(t-\tau)\right\|^{2} d \tau \\
& \leq M^{2} e^{-2 \delta i} e^{2 \delta}\left\|f_{2}\right\|_{S^{2}}^{2} .
\end{aligned}
$$

As a consequence, we get from the Well-Known Weierstrass test that the series $\sum_{i=1}^{\infty} Y_{i}(t)$ is uniformly convergent on $\mathbb{R}$, what is more

$$
Y(t)=\int_{-\infty}^{t} T(\tau) f_{2}(t-\tau) d W(\tau)=\sum_{i=1}^{\infty} Y_{i}(t) \in B C\left(\mathbb{R}, L^{2}(P, \mathbb{H})\right) .
$$

Now, let's shows that

$$
\lim _{T \rightarrow \infty} \frac{1}{m(T, \rho)} \int_{-T}^{T} E\left\|y_{i}(t)\right\|^{2} \rho(t) d t=0
$$


We have

$$
\begin{array}{rl}
\lim _{T \rightarrow \infty} \frac{1}{m(T, \rho)} \int_{-T}^{T} & E\left\|\Phi_{i}(t)\right\|^{2} \rho(t) d t \\
& \leq M^{2} e^{-2 \delta i} e^{2 \delta} \lim _{T \rightarrow \infty} \frac{1}{m(T, \rho)} \int_{-T}^{T} \int_{t-i}^{t-i+1} E\left\|f_{2}(\tau)\right\|^{2} d \tau \\
& \leq M^{2} e^{-2 \delta i} e^{2 \delta} \lim _{T \rightarrow \infty} \frac{1}{m(T, \rho)} \int_{-T}^{T}\left\|f_{2}^{b}(t-i)\right\|_{L^{\infty}\left(\mathbb{R}, L^{2}\right)} d t
\end{array}
$$

Since $f_{2}^{b}(.) \in P A P_{0}\left(\mathbb{R}, L^{2}(P, \mathbb{H}), \rho\right)$, then

$$
\lim _{T \rightarrow \infty} \frac{1}{m(T, \rho)} \int_{-T}^{T}\left\|f_{2}^{b}(t-i)\right\|_{L^{\infty}\left(\mathbb{R}, L^{2}\right)} d t=0 .
$$

Therefore, $Y_{i}(.) \in P A P_{0}\left(\mathbb{R}, L^{2}(P, \mathbb{H}), \rho\right)$, by Lemma (3.2), we get $Y(.) \in P A P_{0}\left(\mathbb{R}, L^{2}(P, \mathbb{H}), \rho\right)$. Finally, $F(.) \in P A P\left(\mathbb{R}, L^{2}(P, \mathbb{H}), \rho\right)$.

Theorem 4.4. Suppose that $\left(\mathbb{A}_{1}-\mathbb{A}_{3}\right)$ hold. If

$$
K=3\left[K_{g}+\frac{M^{2}}{\delta^{2}} K_{h}+\frac{M^{2}}{\delta^{2}} K_{f}\right]<1,
$$

then, the Eq.(1) has a unique weighted pseudo almost periodic solution.

Proof. Define

$$
\begin{aligned}
(\Lambda X)(t) & =g(t, X(t))+\int_{-\infty}^{t} T(t-\tau) h(\tau, X(\tau)) d \tau \\
& +\int_{-\infty}^{t} T(t-\tau) f(\tau, X(\tau)) d W(\tau)
\end{aligned}
$$

$\Lambda$ it is a nonlinear operator on $B C\left(\mathbb{R}, L^{2}(P, \mathbb{H})\right)$, let $X(.) \in P A P\left(\mathbb{R}, L^{2}(P, \mathbb{H}), \rho\right)$, its follows from $\left(A_{1}-A_{3}\right)$ and Lemmas (3.3), (3.11) that $g(., X().) \in P A P\left(\mathbb{R}, L^{2}(P, \mathbb{H}), \rho\right)$ and $h(., X()),. f(., X().) \in P A P S^{2}\left(\mathbb{R}, L^{2}(P, \mathbb{H}), \rho\right)$. It is easy to verify that $h(., X()),. f(., X().) \in$ $C\left(\mathbb{R}, L^{2}(P, \mathbb{H})\right)$. By applying Lemma(4.2) and Lemma(4.3) for $h(., X()$.$) and f(., X()$.$) , we$ deduce that $G(),. \Phi(.) \in P A P\left(\mathbb{R}, L^{2}(P, \mathbb{H}), \rho\right)$. Moreover,

$$
G(t)=\int_{-\infty}^{t} T(t-\tau) h(\tau, X(\tau)) d \tau,
$$

and

$$
\Phi(t)=\int_{-\infty}^{t} T(t-\tau) f(\tau, X(\tau)) d \tau .
$$


We can conclude from the above that the operator $\Lambda$ is defined in $P A P\left(\mathbb{R}, L^{2}(P, \mathbb{H}), \rho\right)$ into itself. Now, let's prove that $\Lambda$ has a unique fixed-point. Let $X, Y \in P A P\left(\mathbb{R}, L^{2}(P, \mathbb{H}), \rho\right)$, we have

$$
\begin{aligned}
E\|(\Lambda X)(t)-(\Lambda Y)(t)\|^{2} & \leq E \| g(t, X(t))-g(t, Y(t)) \\
& +\int_{-\infty}^{t} T(t-\tau)[h(\tau, X(\tau))-h(\tau, Y(\tau))] d \tau \\
& +\int_{-\infty}^{t} T(t-\tau)[f(\tau, X(\tau))-f(\tau, Y(\tau))] d W(\tau) \|^{2}
\end{aligned}
$$

Since $(a+b+c)^{2} \leq 3 a^{2}+3 b^{2}+3 c^{2}$, then

$$
\begin{aligned}
E\|(\Lambda X)(t)-(\Lambda Y)(t)\|^{2} & \leq 3 E\|g(t, X(t))-g(t, Y(t))\|^{2} \\
& +3 E\left\|\int_{-\infty}^{t} T(t-\tau)[h(\tau, X(\tau))-h(\tau, Y(\tau))] d \tau\right\|^{2} \\
& +3 E\left\|\int_{-\infty}^{t} T(t-\tau)[f(\tau, X(\tau))-f(\tau, Y(\tau))] d W(\tau)\right\|^{2},
\end{aligned}
$$

we evaluated the first term on the right side

$$
\begin{aligned}
3 E\|g(t, X(t))-g(t, Y(t))\|^{2} & \leq 3 K_{g} E\|X(t)-Y(t)\|^{2} \\
& \leq 3 K_{g} \sup _{s \in \mathbb{R}} E\|X(s)-Y(s)\|^{2} \\
& \leq 3 K_{g} E\|X-Y\|_{\infty}^{2}
\end{aligned}
$$

For the second term we have

$$
\begin{aligned}
3 \| \int_{-\infty}^{t} T(t-\tau) & {[h(\tau, X(\tau))-h(\tau, Y(\tau))] d \tau \|^{2} } \\
& \leq 3 E\left(\int_{-\infty}^{t}\|T(t-\tau)\|\|h(\tau, X(\tau))-h(\tau, Y(\tau))\| d \tau\right)^{2} \\
& \leq 3 M^{2} E\left(\int_{-\infty}^{t} e^{-\delta(t-\tau)}\|h(\tau, X(\tau))-h(\tau, Y(\tau))\| d \tau\right)^{2} \\
& \leq 3 M^{2}\left(\int_{-\infty}^{t} e^{-\delta(t-\tau)} d \tau\right)\left(\int_{-\infty}^{t} e^{-\delta(t-\tau)} E\|h(\tau, X(\tau))-h(\tau, Y(\tau))\|^{2} d \tau\right) \\
& \leq 3 M^{2} K_{h}\left(\int_{-\infty}^{t} e^{-\delta(t-\tau)} d \tau\right)\left(\int_{-\infty}^{t} e^{-\delta(t-\tau)} E\|X(\tau)-Y(\tau)\|^{2} d \tau\right) \\
& \leq \frac{3 M^{2}}{\delta^{2}} K_{h}\|X-Y\|_{\infty}^{2} .
\end{aligned}
$$


As to the last term, we use an estimate on the Ito integral ( [18])

$$
\begin{aligned}
3 E \| \int_{-\infty}^{t} T(t-\tau) & {[f(\tau, X(\tau))-f(\tau, Y(\tau))] d W(\tau) \|^{2} } \\
& \leq 3 E\left[\int_{-\infty}^{t}\|T(t-\tau)[f(\tau, X(\tau))-f(\tau, Y(\tau))]\|^{2} d(\tau)\right] \\
& \leq 3 E\left[\int_{-\infty}^{t}\|T(t-\tau)\|^{2}\|f(\tau, X(\tau))-f(\tau, Y(\tau))\|^{2} d(\tau)\right] \\
& \leq 3 M^{2} \int_{-\infty}^{t} e^{-2 \delta(t-\tau)} E\|f(\tau, X(\tau))-f(\tau, Y(\tau))\|^{2} d(\tau) \\
& \leq 3 M^{2} K_{f} \int_{-\infty}^{t} e^{-2 \delta(t-\tau)} E\|X(\tau)-Y(\tau)\|^{2} d(\tau) \\
& \leq 3 M^{2} K_{f} \int_{-\infty}^{t} e^{-2 \delta(t-\tau)} d \tau \quad \underset{s \in \mathbb{R}}{\sup } E\|X(\tau)-Y(\tau)\|^{2} \\
& \leq \frac{3 M^{2}}{2 \delta} K_{f}\|X-Y\|_{\infty}^{2} .
\end{aligned}
$$

By combining all inequality together, we get

$$
E\|(\Lambda X)(t)-(\Lambda Y)(t)\|^{2} \leq 3\left[K_{g}+\frac{M^{2}}{\delta^{2}} K_{h}+\frac{M^{2}}{\delta^{2}} K_{f}\right]\|X-Y\|_{\infty}^{2} .
$$

Therefore,

$$
E\|\Lambda X-\Lambda Y\|_{\infty} \leq \sqrt{K}\|X-Y\|_{\infty}
$$

We have $\sqrt{K}<1$, then the operator $\Lambda$ is a strict contractions, so by using the Banach contraction principal we come to the conclusion that there exist a unique fixed point $X($.$) for \Lambda$. To complete the proof, it suffices to prove that $\mathrm{X}$ satisfies(1). For all $s \in \mathbb{R}$ and $t \geq s$, we get

$$
\begin{aligned}
X(s)-g(s, X(s)) & =\int_{-\infty}^{s} T(s-\tau) h(\tau, X(\tau)) d \tau \\
& +\int_{-\infty}^{s} T(s-\tau) f(\tau, X(\tau)) d W(\tau) .
\end{aligned}
$$

Multiply both sides by $T(t-s)$, then

$$
\begin{aligned}
T(t-s) & (X(s)-g(s, X(s))) \\
& =T(t-s) \int_{-\infty}^{s} T(s-\tau) h(\tau, X(\tau)) d \tau+T(t-s) \int_{-\infty}^{s} T(s-\tau) f(\tau, X(\tau)) d W(\tau) \\
& =\int_{-\infty}^{s} T(t-\tau) h(\tau, X(\tau)) d \tau+\int_{-\infty}^{s} T(t-\tau) f(\tau, X(\tau)) d W(\tau) \\
& =\int_{-\infty}^{t} T(t-\tau) h(\tau, X(\tau)) d \tau+\int_{-\infty}^{t} T(t-\tau) f(\tau, X(\tau)) d W(\tau)
\end{aligned}
$$




$$
\begin{aligned}
& -\int_{s}^{t} T(t-\tau) h(\tau, X(\tau)) d \tau-\int_{s}^{t} T(t-\tau) f(\tau, X(\tau)) d W(\tau) \\
& =X(t)-g(t, X(t))-\int_{s}^{t} T(t-\tau) h(\tau, X(\tau)) d \tau \\
& -\int_{s}^{t} T(t-\tau) f(\tau, X(\tau)) d W(\tau) .
\end{aligned}
$$

Finally, $X \in P A P\left(\mathbb{R}, L^{2}(P, \mathbb{H}), \rho\right)$ is a unique mild solution to equation (1).

\section{Example}

We consider the following neutral partial stochastic differential equation

$$
\left\{\begin{array}{l}
d X(t, s)=\frac{\partial^{2}}{\partial x^{2}} X(t, s) d t+d g(t, X(t, s))+h(t, X(t, s)) d t+g(t, X(t, s)) d W(t),(t, s) \in \mathbb{R} \times(0,1) . \\
X(t, 0)=X(t, 1)=0 .
\end{array}\right.
$$

Where

$$
\begin{gathered}
g(t, X)=\frac{1}{18}\left((\sin (t)+\sin (\sqrt{2} t)) X+e^{-|t|} \sin (X)\right) . \\
h(t, X)=\frac{1}{5}(\cos (2 \pi t)+\cos (t)) X+\frac{1}{8} e^{-|t|} \cos (X) . \\
f(t, X)=\frac{1}{6}(\sin (2 \pi \sqrt{2} t)+\sin (2 \pi t)) X+\frac{1}{3} e^{-|t|} \cos (X) .
\end{gathered}
$$

Let $\left(\Omega, \mathbf{F},\left(\mathbf{F}_{t}\right)_{t \geq 0}, P\right)$ the filtered probability space, $(W(t), t \in \mathbb{R})$ is two-sided standard Weiner process on $L^{2}(0,1)$ and $\rho(t)=\frac{3}{2}\left(1+t^{2}\right)$, it is easy to see that $m(T, \rho)=\int_{-T}^{T} \rho(t) d t=3 T+T^{3}$, then $\rho \in \mathcal{U}_{\infty}^{i n v}$.

In order to write system (2) on the abstract (1), we consider the following linear operator $A: D(A) \subset L^{2}(0,1) \rightarrow L^{2}(0,1)$, where

$$
\begin{gathered}
D(A)=H^{2}(0,1) \cap H_{0}^{1}(0,1) . \\
A x(s)=x^{\prime \prime}(s) \quad s \in(0,1) \text { and } x \in D(A) .
\end{gathered}
$$

$A$ generates a $C_{0}$-semigroup $\left(T(t)_{t \geqslant 0}\right)$ on $L^{2}(0,1)$, which is specified by

$$
(T(t) x)(r)=\sum_{n=1}^{\infty} e^{-n^{2} \pi^{2} t}<x, e_{n}>_{L^{2}} e_{n}(r)
$$

where $e_{n}(r)=\sqrt{2} \sin (n \pi r)$ for $n=1,2, \ldots$, and $\|T(t)\| \leq e^{-\pi^{2} t} \forall t \geq 0$, we set $M=1$, $\delta=\pi^{2}$ and $H=L^{2}([0,1])$. it is easy to show that $\frac{1}{18}(\sin (t)+\sin (\sqrt{2} t)) X$ belong to 
$A P\left(\mathbb{R} \times L^{2}(P, \mathbb{H}), L^{2}(P, \mathbb{H})\right)$. Since,

$$
\begin{aligned}
\frac{1}{m(T, \rho)} \int_{0}^{T} E\left\|e^{-|t|} \sin (X)\right\|^{2} \rho(t) d t & \\
\leq & \frac{1}{3 T+T^{3}} \int_{0}^{T} e^{-2 t} \frac{3}{2}\left(1+t^{2}\right) d t \rightarrow 0 \quad \text { as } \quad T \rightarrow 0 .
\end{aligned}
$$

And,

$$
\begin{aligned}
\frac{1}{m(T, \rho)} \int_{-T}^{0} E\left\|e^{-|t|} \sin (X)\right\|^{2} \rho(t) d t & \\
\leq & \frac{1}{3 T+T^{3}} \int_{-T}^{0} e^{2 t} \frac{3}{2}\left(1+t^{2}\right) d t \rightarrow 0 \quad \text { as } \quad T \rightarrow 0
\end{aligned}
$$

Then, $\frac{1}{18} e^{-|t|} \sin (X)$ belong to $P A P_{0}\left(\mathbb{R} \times L^{2}(P, \mathbb{H}), L^{2}(P, \mathbb{H}), \rho\right)$. So, $g(.,.) \in P A P(\mathbb{R} \times$ $\left.L^{2}(P, \mathbb{H}), L^{2}(P, \mathbb{H}), \rho\right)$. Similar to the argument presented above, we have $h(.,),. f(.,$. $\in P A P\left(\mathbb{R} \times L^{2}(P, \mathbb{H}), L^{2}(P, \mathbb{H}), \rho\right)$, and it is clear that $P A P\left(\mathbb{R} \times L^{2}(P, \mathbb{H}), L^{2}(P, \mathbb{H}), \rho\right) \subset$ $P A P S^{2}\left(\mathbb{R} \times L^{2}(P, \mathbb{H}), L^{2}(P, \mathbb{H}), \rho\right)$, then we get $h(.,),. f(.,.) \in P A P S^{2}\left(\mathbb{R} \times L^{2}(P, \mathbb{H}), L^{2}(P, \mathbb{H}), \rho\right)$. Clearly, $g, h$ and $f$ satisfy the Lipschitz conditions $\left(A_{3}\right)$ with $L_{g}=\frac{1}{6}, L_{h}=\frac{21}{40}, L_{f}=\frac{2}{3}$, then

$$
3\left[L_{g}+\frac{M^{2}}{\delta^{2}} L_{h}+\frac{M^{2}}{2 \delta} L_{f}\right]=3\left[\frac{1}{6}+\frac{1}{\pi^{2}} \frac{21}{40}+\frac{1}{2 \pi} \frac{2}{3}\right] \simeq 0.98<1 .
$$

Therefore, by Theorem (4.4) the equation (2) has a unique weighted pseudo almost periodic solution.

\section{REFERENCES}

[1] R.P. Agarwal, T. Diagana and E. Hernandez, Weighted pseudo almost periodic solution to some partial neutral functional differential equations, J. Nonlinear Convex Anal. 8 (2007), 397-415.

[2] T. Diagana, Existence of weighted pseudo almost periodic solutions to some classes of hyperbolic evolution equations, J. Math. Anal. Appl. 350 (2009), 18-28. https://doi .org/10.1016/j . jmaa. 2008.09.041.

[3] T. Diagana, Stepanov-like pseudo-almost periodicity and its applications to some nonautonomous differential equations, Nonlinear Anal.: Theory Methods Appl. 69 (2008), 4277-4285. https : //doi .org/10.1016/ j.na.2007.10.051.

[4] T. Diagana, Weighted pseudo almost periodic functions and applications,C. R. Acad. Sci. Paris, Ser. I. 343 (2006),643-646. https://doi.org/10.1016/j.crma.2006.10.008.

[5] T. Diagana, Weighted pseudo-almost periodic solutions to a neutral delay integral equation of advanced type, Nonlinear Anal.: Theory Methods Appl. 70 (2009), 298-304. https://doi .org/10.1016/j .na. 2007. 11.052.

[6] T. Diagana, Weighted pseudo-almost periodic solutions to some differential equations, Nonlinear Anal.: Theory Methods Appl. 68 (2008), 2250-2260. https://doi.org/10.1016/j . na. 2007.01.054. 
[7] T. Diagana, G.M. Mophou, G.M. N’Guérékata, Existence of weighted pseudo-almost periodic solutions to some classes of differential equations with $S^{p}$-weighted pseudo-almost periodic coefficients, Nonlinear Anal.: Theory Methods Appl. 72 (2010), 430-438. https://doi .org/10.1016/j .na. 2009.06.077.

[8] T. Diagana, M. Zitane, Stepanov-like pseudo-almost periodic functions in lebesgue spaces with variable exponents $L^{p(x)}$, in: B. Toni (Ed.), New Frontiers of Multidisciplinary Research in STEAM-H (Science, Technology, Engineering, Agriculture, Mathematics, and Health), Springer International Publishing, Cham, 2014: pp. 295-314. https://doi.org/10.1007/978-3-319-07755-0_13.

[9] T. Diagana and M. Zitane, Weighted Stepanov-like pseudo almost periodic funcitons in Lebesgue spaces with variable exponents $L^{p(x)}$. Afr. Diaspora J. Math. (N.S.) 15 (2013), 56-75.

[10] M. Es-saiydy, M. Zitane, Weighted Stepanov-like pseudo almost periodicity on time scales and applications, Differ. Equ. Dyn. Syst. (2020). https://doi .org/10.1007/s12591-020-00543-7.

[11] M. Es-saiydy, M. Zitane, Dynamic behavior of a class of delayed Lotka-Volterra recurrent neural networks on time scales, Russ. Math. 65 (2021), 59-75. https : //doi .org/10.3103/S1066369X21110074.

[12] J.K. Hale, Partial neutral functional differential equations, Rev. Roumaine Math. Pures Appl. 39 (1994), 339-344.

[13] J.K. Hale, S.M.V. Lunel, Introduction to functional differential equations, Springer New York, New York, 1993. https://doi.org/10.1007/978-1-4612-4342-7.

[14] E. Hernández, Existence results for partial neutral integro-differential equations with unbounded delay, J. Math. Anal. Appl. 292 (2004), 194-210.

[15] E. Hernández, H.R. Henríquez, Existence of periodic solutions of partial neutral functional differential equations with unbounded delay, J. Math. Anal. Appl. 221 (1998), 499-522. https://doi .org/10.1006/ jmaa.1997.5899.

[16] E. Hernández, H.R. Henríquez, Existence results for partial neutral functional differential equations with unbounded delay, J. Math. Anal. Appl. 221 (1998), 452-475. https://doi .org/10.1006/jmaa.1997. 5875.

[17] Z. Hu, Z. Jin, Stepanov-like pseudo-almost periodic mild solutions to perturbed nonautonomous evolution equations with infinite delay, Nonlinear Anal.: Theory Methods Appl. 71 (2009), 5381-5391. https://doi. org/10.1016/j.na.2009.04.032.

[18] A. Ichikawa, Stability of semilinear stochastic evolution equations, J. Math. Anal. Appl. 90 (1982), $12-44$. https://doi.org/10.1016/0022-247X (82)90041-5.

[19] J. Wu, Theory and applications of partial functional differential equations, Springer New York, New York, NY, 1996. https://doi .org/10.1007/978-1-4612-4050-1.

[20] J. Wu and H. Xia, Rotating waves in neutral partial functional-differential equations, J. Dyn. Differ. Equ. 11 (1999), 209-238. https://doi.org/10.1023/A:1021973228398.

[21] J. Wu, H. Xia, Self-sustained oscillations in a ring array of coupled lossless transmission lines, J. Differ. Equ. 124 (1996), 247-278. https://doi.org/10.1006/jdeq.1996.0009.

[22] Z. Yan, H. Zhang, Existence of Stepanov-like square-mean pseudo almost periodic solutions to partial stochastic neutral differential equations, Ann. Funct. Anal. 6 (2015), 116-138. https://doi .org/10.15352/ afa/06-1-10. 
[23] S. Zaidman, Almost-periodic functions in abstract spaces, Pitman Advanced Pub. Program, Boston, 1985.

[24] C.Y. Zhang, Integration of vector-valued pseudo-almost periodic functions, Proc. Amer. Math. Soc. 121 (1994),167-174. https://doi.org/10.1090/s0002-9939-1994-1186140-8.

[25] C.Y. Zhang, Pseudo almost periodic solutions of some differential equations, J. Math. Anal. Appl. 181 (1994),62-76. https://doi.org/10.1006/jmaa.1994.1005.

[26] C.Y. Zhang, Pseudo almost periodic solutions of some differential equations, II, J. Math. Anal. Appl. 192 (1995), 543-561. https://doi.org/10.1006/jmaa.1995.1189.

[27] L. Zhang and Y. Xu, Weighted pseudo almost periodic solution for functional differential equations, Electron. J. Differ. Equ. 2007 (2007), 146.

[28] L. Zhang, Y. Xu, Weighted pseudo-almost periodic solutions of a class of abstract differential equations, Nonlinear Anal.: Theory Methods Appl. 71 (2009),3705-3714. https://doi .org/10.1016/j .na.2009.02. 032. 